\title{
Cold plasma dispersion surfaces
}

\author{
By M. E. OAKES, R. B. MICHIE, K. H. TSUI
}

Department of Physics, The University of Texas, Austin, Texas 78712

\author{
AND J. E. COPELAND
}

Department of Physics, Southwestern University, Georgetown, Texas 78726

(Received 5 July 1978)

Three-dimensional plots of dispersion in a cold anisotropic plasma are presented. The $\omega(k, \theta)$ surfaces provide a clear picture of the behaviour of cold plasma waves as the direction of propagation is varied. The group velocity $(d \omega / d \mathbf{k})$ has a simple geometrical interpretation on the surfaces.

\section{Introduction}

In Stix's (1962) excellent book on plasma waves, he presented the theory of cold plasma waves using a diagram first proposed by Clemmow \& Mullaly (1955) and later modified by Allis (1959) and Allis, Buchsbaum \& Bers (1962). This CMA diagram displays the behaviour of the phase velocity with direction of propagation with respect to a static magnetic field. Plots of the wave normal surfaces are given in a plasma parameter space. A surface is shown to remain topologically equivalent provided one remains in one of 13 regions in the parameter space. The CMA diagram is particularly useful for studying cut-offs, resonances, accessibility and other inhomogeneous plasma effects (provided the density scale length is large compared with the plasma wavelength).

A second important contribution was made by Stringer (1963) who introduced thermal effects using scalar pressures and plotted $\omega$ versus $k$, the wave vector for a fixed angle of propagation. He included only the low-frequency waves. Each portion of the dispersion curve is accompanied by very helpful physical pictures of the dominant effects contributing to the waves. Stringer chose angles of propagation of $45^{\circ}$ and nearly perpendicular to the static magnetic field. The inclusion of pressure effects gives rise to an additional branch, modifies resonances and cut-offs and alters phase velocities.

In this paper we present the cold plasma modes along the lines of Stringer's dispersion curves; however we include all angles of propagation and thus generate surfaces. These surfaces are plots of $\omega$ versus $k$ and $\theta$. Using the surfaces one is able to follow the behaviour of waves as the direction of propagation is varied. It will be seen that the principal waves $(\theta=0$ and $\theta=\pi / 2)$

* Present address: Instituto de Física, Universidade Federal Fluminense, Outeiro de São João Batista S/no., Campus Universitario, Niteroi, Rio de Janeiro, 24000, Brasil. 
usually presented in elementary plasma text result from contributions by two or more surfaces. The group velocity is seen to have a simple geometrical interpretation.

\section{Dispersion relation}

The dispersion relation for a cold collisionless plasma is fourth order in $k$ (Allis 1959) which yields two distinct modes of propagation, the other two being oppositely directed waves. The dispersion relation is tenth order in $\omega$, indicating five distinct surfaces in a plot of $\omega$ versus $k$ and $\theta$, where $k$ is the magnitude of the wave vector and $\theta$ is the angle between $\mathbf{k}$ and the static magnetic field. The dispersion relation is

$$
\begin{aligned}
& \omega^{10}-\omega^{8}\left[2 c^{2} k^{2}+\Omega_{e}^{2}+\Omega_{i}^{2}+3\left(\omega_{p e}^{2}+\omega_{i p}^{2}\right)\right]+\omega^{6}\left[c^{4} k^{4}+2 c^{2} k^{2}\left(\Omega_{e}^{2}+\Omega_{i}^{2}\right.\right. \\
& \left.\left.\quad+2\left(\omega_{p e}^{2}+\omega_{p i}^{2}\right)\right)+\left(\Omega_{e} \Omega_{i}+\omega_{p e}^{2}+\omega_{p i}^{2}\right)^{2}+\left(\omega_{p e}^{2}+\omega_{i p}^{2}\right)\left(2\left(\omega_{p e}^{2}+\omega_{p i}^{2}\right)+\Omega_{e}^{2}+\Omega_{i}^{2}\right)\right] \\
& \quad-\omega^{4}\left[c^{4} k^{4}\left(\omega_{p e}^{2}+\omega_{p i}^{2}+\Omega_{e}^{2}+\Omega_{i}^{2}\right)+2 c^{2} k^{2}\left(\omega_{p e}^{2}+\omega_{p i}^{2}+\Omega_{e} \Omega_{i}\right)^{2}+c^{2} k^{2}\left(1+\cos ^{2} \theta\right)\right. \\
& \left.\quad\left(\omega_{p e}^{2}+\omega_{p i}^{2}\right)\left(\Omega_{e}^{2}+\Omega_{i}^{2}-\Omega_{e} \Omega_{i}\right)+\left(\omega_{p e}^{2}+\omega_{p i}^{2}\right)\left(\Omega_{e} \Omega_{i}+\omega_{p e}^{2}+\omega_{p i}^{2}\right)^{2}\right] \\
& \quad+\omega^{2}\left[c^{4} k^{4}\left\{\left(\omega_{p e}^{2}+\omega_{p i}^{2}\right)\left(\Omega_{e}^{2}+\Omega_{i}^{2}\right) \cos ^{2} \theta+\Omega_{e} \Omega_{i} \sin ^{2} \theta+\Omega_{e}^{2} \Omega_{i}^{2}\right\}\right. \\
& \left.\quad+c^{2} k^{2}\left(\left(\omega_{p e}^{2}+\omega_{p i}^{2}\right) \Omega_{e} \Omega_{i}\left(\Omega_{e} \Omega_{i}+\omega_{p e}^{2}+\omega_{p i}^{2}\right)\left(1+\cos ^{2} \theta\right)\right)\right] \\
& \quad-\Omega_{e}^{2} \Omega_{i}^{2}\left(\omega_{p e}^{2}+\omega_{p i}^{2}\right) c^{4} k^{4} \cos ^{2} \theta=0
\end{aligned}
$$

where

$$
\begin{aligned}
\omega_{p e}^{2} & =4 \pi n e^{2} / m_{e}, \quad \omega_{p i}^{2}=4 \pi n Z e^{2} / M_{i}, \quad \omega_{p}^{2}=\omega_{p e}^{2}+\omega_{p i}^{2}, \\
\Omega_{e} & =e B / m_{e} c, \quad \Omega_{i}=e B / M_{i} c, \\
\tan \theta & =k_{\perp} / k_{\|}, \quad k^{2}=k_{\perp}^{2}+k_{\|}^{2} .
\end{aligned}
$$

Additional important frequencies and quantities are defined at this point:

Upper and lower hybrid frequencies $\left(\omega_{U H}, \omega_{L H}\right)$

$$
\begin{aligned}
\omega_{U H}^{2}+\omega_{L H}^{2} & =\omega_{p}^{2}+\Omega_{e}^{2}+\Omega_{i}^{2}, \\
\omega_{U H}^{2} \omega_{L H}^{2} & =\Omega_{e} \Omega_{i}\left(\omega_{p}^{2}+\Omega_{e} \Omega_{i}\right) .
\end{aligned}
$$

Cut-off frequencies $\left(\omega_{R}, \omega_{L}\right)$

$$
\omega_{R}^{2} \mp \omega_{R}\left(\Omega_{e}-\Omega_{i}\right)-\omega_{p e}^{2}-\omega_{p i}^{2}-\Omega_{e} \Omega_{i}=0 .
$$

Stix parameters $\left(R, L, P, \theta_{r}\right)$

$$
\begin{aligned}
& R=1-\frac{\omega_{p e}^{2}}{\omega\left(\omega-\Omega_{e}\right)}-\frac{\omega_{p i}^{2}}{\omega\left(\omega+\Omega_{i}\right)}, \\
& L=1-\frac{\omega_{p e}^{2}}{\omega\left(\omega+\Omega_{e}\right)}-\frac{\omega_{p i}^{2}}{\omega\left(\omega-\Omega_{i}\right)}, \\
& P=1-\frac{\omega_{p}^{2}}{\omega^{2}}, \quad \tan ^{2} \theta_{r}=-\frac{2 P}{R+L} .
\end{aligned}
$$




\section{Dispersion surfaces}

The surfaces are first presented on a linear scale with principal waves at $\theta=0$ and $\theta=\pi / 2$ labelled. We start with the highest frequency surface and proceed to the lowest. Plasma conditions are such that $\Omega_{e}^{2}>\omega_{p e}^{2}$. In figure 1 we see a perspective surface which has a cut-off at $\omega_{R}$. For propagation along the magnetic field $(\theta=0)$ the wave is right-hand circularly polarized ( $R$-wave) and proceeds from the cut-off to the light cone $(\omega=k c)$. At $\theta=\pi / 2$, the extraordinary wave ( $X$-wave) starts at $\omega_{R}$ and asymptotes to the light cone. A number of constant $\omega$ contours are shown proceeding from $C_{1}$, the lowest, to $C_{6}$, the largest; $k_{\|}$and $k_{\perp}$ are the propagation vector components parallel and perpendicular to the static magnetic field.

In figure 2 we show the contour lines of figure 1 . As the light cone is approached, the contours become circular as expected. The lowering of the phase velocity as propagation is directed more nearly perpendicular to the magnetic field should be noted.

Figure 3 shows a second high frequency surface with a cut-off at $\omega^{2}=\omega_{p e}^{2}+\omega_{p i}^{2}$. Again for high frequencies the surface becomes the light cone. Along the magnetic field this surface contains a portion of the left-hand circularly polarized wave $\left(n^{2}=L\right.$ in the Stix notation, where $n$ is the refractive index) and also part of the non-dispersive, zero group velocity plasma oscillation $P=0\left(\omega^{2}=\omega_{p e}^{2}+\omega_{p i}^{2}\right)$. At $\theta=\pi / 2$ this is the ordinary wave $\left(n^{2}=P\right)$ which is linearly polarized with $\mathbf{E}_{\|} \mathbf{B}_{0}$. It is seen that the ordinary wave is relatively insensitive to small additions of $k_{1}$, a property highly desirable for microwave interferometric measurements. The contours are similar to those in figure 2 except in the neighbourhood of $\omega_{p}$.

Figure 4 shows a surface containing a cut-off $\left(\omega_{L}\right)$ and resonances. At $\theta=0$ this surface describes three types of waves. Between $\omega_{L}$ and $\omega_{p}$ the wave is left-hand circularly polarized; at $\omega_{p}$ the surface flattens and contributes a small amount to the plasma oscillations $(P=0)$. Above $\omega_{p}$ the wave is right-hand circularly polarized and approaches a resonance at $\Omega_{e}$. Perpendicular to the field $(\theta=\pi / 2)$, one has the extraordinary wave which has a cut-off at $\omega_{L}$ and approaches a resonance at the upper hybrid frequency $\omega_{U H}$ (where

$$
\left.\omega_{U H}^{2} \simeq \omega_{p e}^{2}+\Omega_{e}^{2}\right) .
$$

Note the scale change on $\omega, k_{\|}$and $k_{\perp}$. Figure 5 is a contour plot of figure 4 . The values of $k_{\perp}$ and $k_{\|}$are not large enough to show the contour lines approaching the resonant angle $\theta_{r}$. This behaviour will be seen later in the log plots.

In figure 6 we see the surface which at $\theta=0$ includes the right-hand circularly polarized wave $\left(n^{2}=R\right)$ between $0<\omega<\omega_{p}$; at $\omega=\omega_{p}$ this surface includes the plasma oscillation line $(P=0)$. If the plasma frequency were larger than the electron cyclotron frequency then the right-hand wave would resonate at $\Omega_{e}$. At $\theta=\pi / 2$ we see the portion of the extraordinary wave which includes the lower hybrid resonance $\omega_{L H}^{2} \simeq \Omega_{e} \Omega_{i}\left(\omega_{p e}^{2}+\Omega_{e} \Omega_{i}\right) /\left(\omega_{p e}^{2}+\Omega_{e}^{2}\right)$. The Whistler and compressional Alfvén region of the surface is shown. (The low frequency portion of the surface is expanded in figure 8.) The contour plots in figure 7 show clearly the 


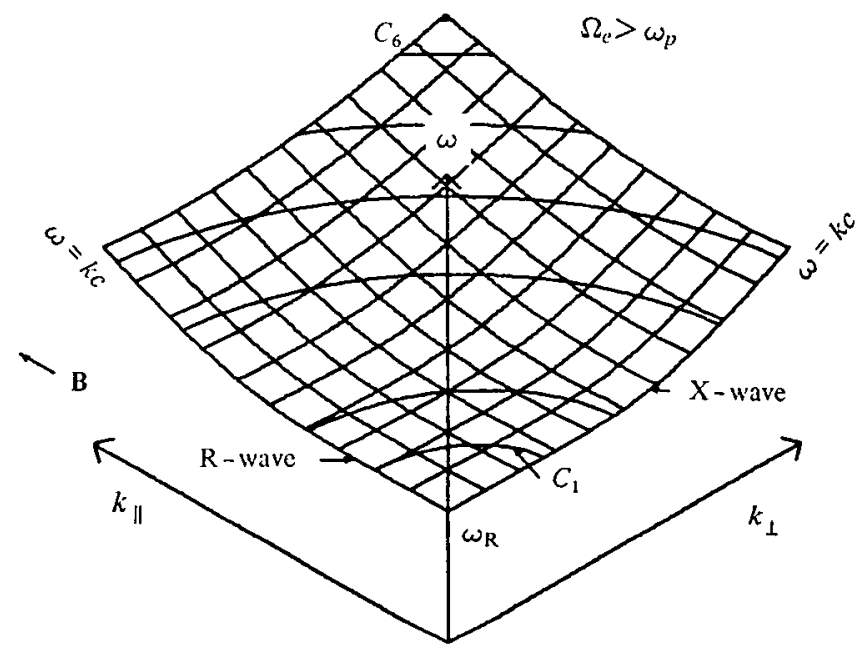

Frgure 1. High frequency $R, X$ surface. $\omega$ vs. $k_{1}$ and $k_{\perp}$. Perspective plot. Linear scale. $B_{0}=100 \mathrm{kG}, n_{0}=10^{14} \mathrm{~cm}^{-8}, 0 \leqslant k_{1} \leqslant 100 \mathrm{~cm}^{-1}, 0 \leqslant k_{1} \leqslant 100 \mathrm{~cm}^{-1}, 0 \leqslant \omega \leqslant 6 \times 10^{12} \mathrm{sec}^{-1}$. Constant $\omega$ contours: $C_{1}=1.94 \times 10^{12} \mathrm{sec}^{-1}, C_{2}=2 \times 10^{12} \mathrm{sec}^{-1}, C_{8}=2.5 \times 10^{12} \mathrm{sec}^{-1}$, $C_{4}=3 \times 10^{12} \mathrm{sec}^{-1}, C_{5}=3.5 \times 10^{12} \mathrm{sec}^{-1}, C_{6}=4.0 \times 10^{18} \mathrm{sec}^{-1}, C_{7}=4.25 \times 10^{18} \mathrm{sec}^{-1}$.

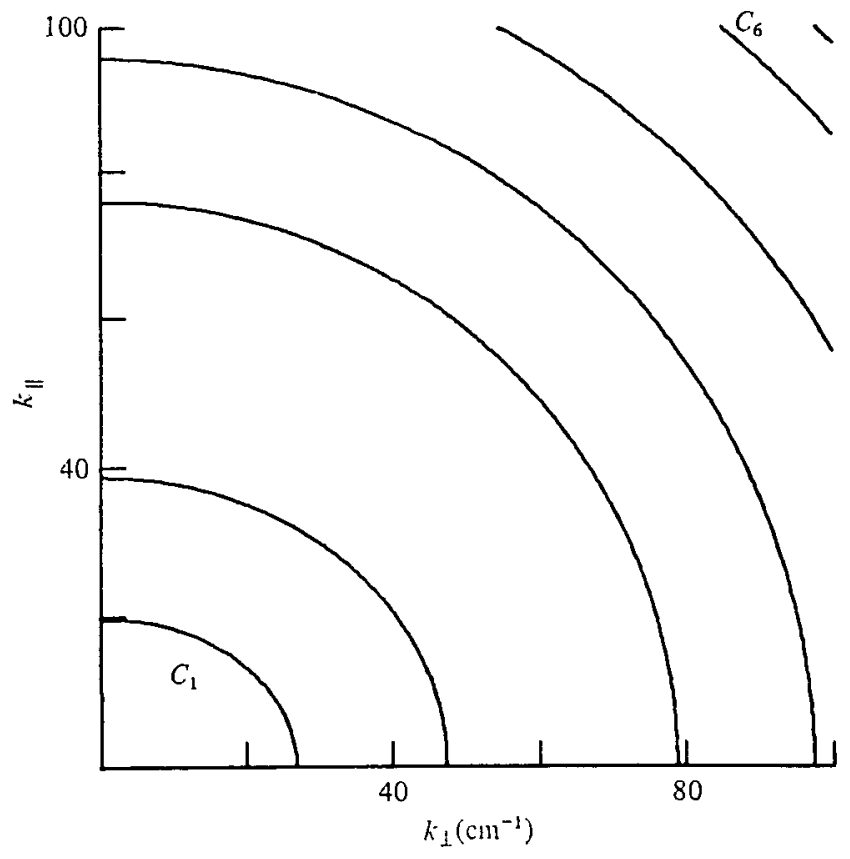

FIGURE 2. Contours of high frequency $R, X$ surface.

Contour values are the same as in figure 1. 


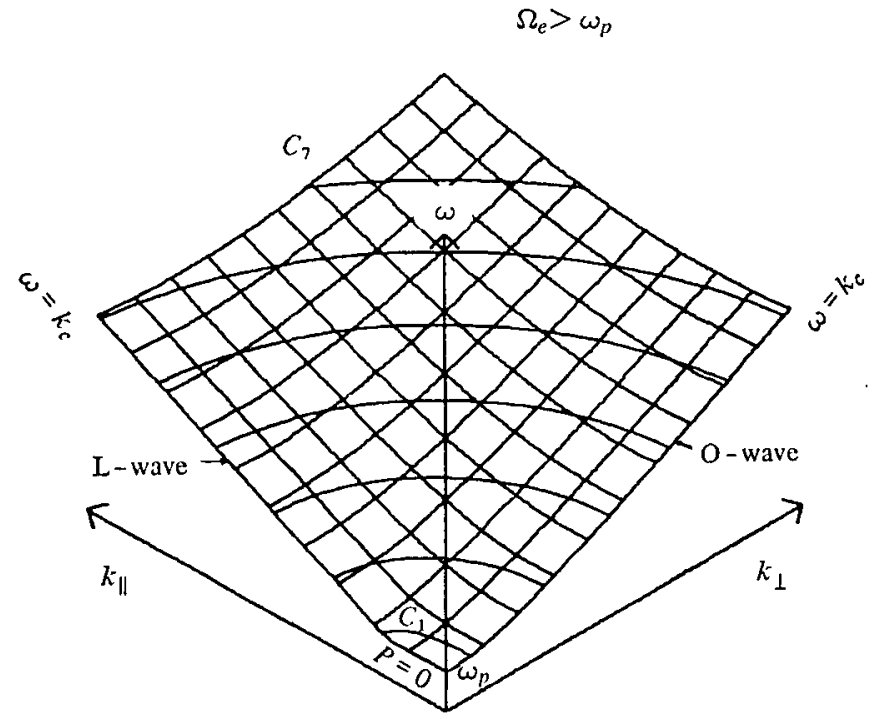

Figure 3. High frequency $L, O$ surface. $\omega$ vs. $k_{n}$ and $k_{\perp}$. Perspective plot. Linear scale. $B_{0}=100 \mathrm{kG}, n_{e}=10^{14} \mathrm{~cm}^{-3}, 0 \leqslant k_{1} \leqslant 100 \mathrm{~cm}^{-1}, 0 \leqslant k_{\perp} \leqslant 100 \mathrm{~cm}^{-1}$. Constant $\omega$ contours are the same as in figure 1 .

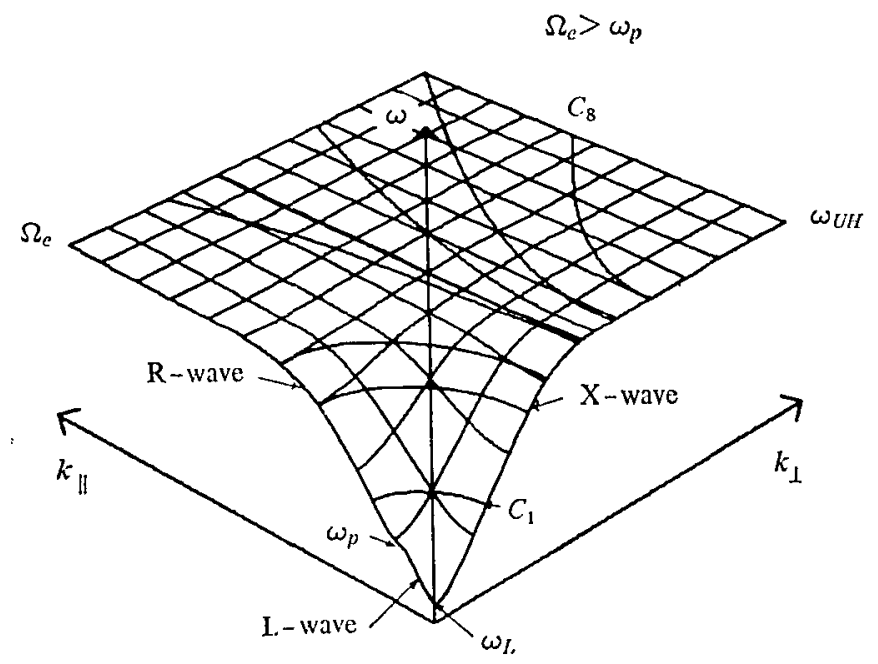

Figure 4. Electron cyclotron-upper hybrid surface. $\omega$ vs. $k_{\mathrm{a}}$ and $k_{\perp}$. Perspective plot. Linear scale. $B_{0}=100 \mathrm{kG}, n_{s}=10^{14} \mathrm{~cm}^{-3}, 0 \leqslant k_{1} \leqslant 200 \mathrm{~cm}^{-1}, 0 \leqslant k_{\perp} \leqslant 200 \mathrm{~cm}^{-1}$,

$$
0 \leqslant \omega \leqslant 4 \times 10^{12} \mathrm{sec}^{-1} \text {. }
$$

Constant $\omega$ contours: $C_{1}=8 \times 10^{11} \mathrm{sec}^{-1}, C_{2}=1.4 \times 10^{12} \mathrm{sec}^{-1}, C_{3}=1.6 \times 10^{12} \mathrm{sec}^{-1}$, $C_{4}=1.75 \times 10^{12} \mathrm{sec}^{-1}, C_{5}=1.759 \times 10^{12} \mathrm{sec}^{-1}, C_{6}=1.78 \times 10^{12} \mathrm{sec}^{-1}, C_{7}=1.8 \times 10^{12} \mathrm{sec}^{-1}$, $C_{8}=1.82 \times 10^{12} \mathrm{sec}^{-1}, C_{9}=1.84 \times 10^{12} \mathrm{sec}^{-1}$. 


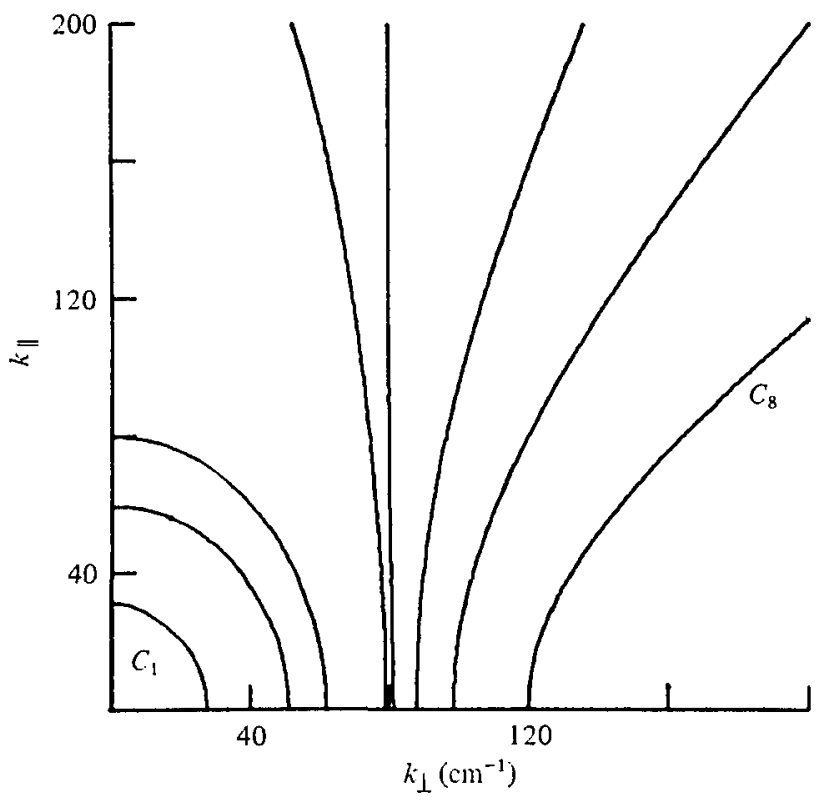

Fradre 5. Contours of electron cyclotron-upper hybrid surface. Contour values are the same as in figure 4.

$$
\Omega_{c}>\omega_{p}
$$

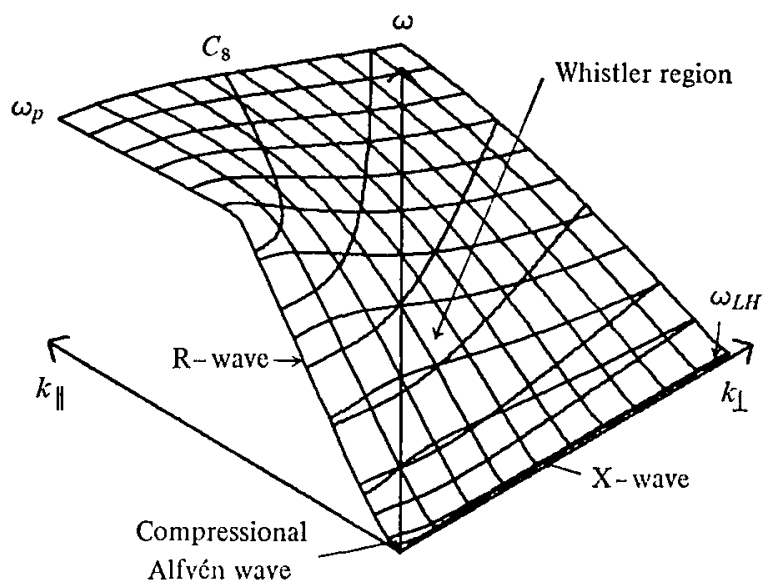

Fraure 6. Compressional Alfvén-lower hybrid surface. $\omega$ vs. $k_{\|}$and $k_{\perp}$. Perspective plot. Linear scale. $B_{0}=100 \mathrm{kG}, n_{e}=10^{14} \mathrm{~cm}^{-3}, 0 \leqslant k_{n} \leqslant 50 \mathrm{~cm}^{-1}, 0 \leqslant k_{\perp} \leqslant 50 \mathrm{~cm}^{-1}$, $0 \leqslant \omega \leqslant 10^{12} \mathrm{sec}^{-1}$. Constant $\omega$ contours: $C_{1}=5 \times 10^{9} \mathrm{sec}^{-1}, C_{2}=1.257 \times 10^{10} \mathrm{sec}^{-1}, C_{3}=$ $5 \times 10^{10} \mathrm{sec}^{-1}, C_{4}=10^{11} \mathrm{sec}^{-1}, C_{5}=2 \times 10^{11} \mathrm{sec}^{-1}, C_{6}=3 \times 10^{11} \mathrm{sec}^{-1}, C_{7}=4 \times 10^{11} \mathrm{sec}^{-1}$, $C_{8}=5 \times 10^{11} \mathrm{sec}^{-1}$. 


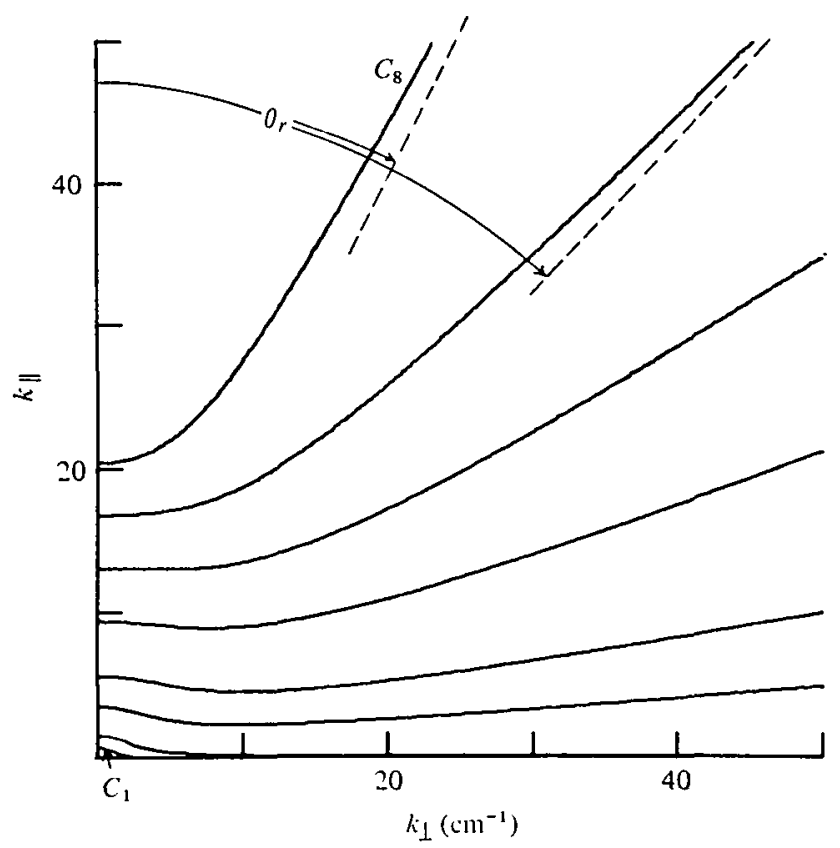

FigURE 7. Contours of compressional Alfvén-lower hybrid surface.

Contour values are the same as in figure 6. $\theta_{r}=\tan ^{-1}(-P / S)^{\text {t. }}$.

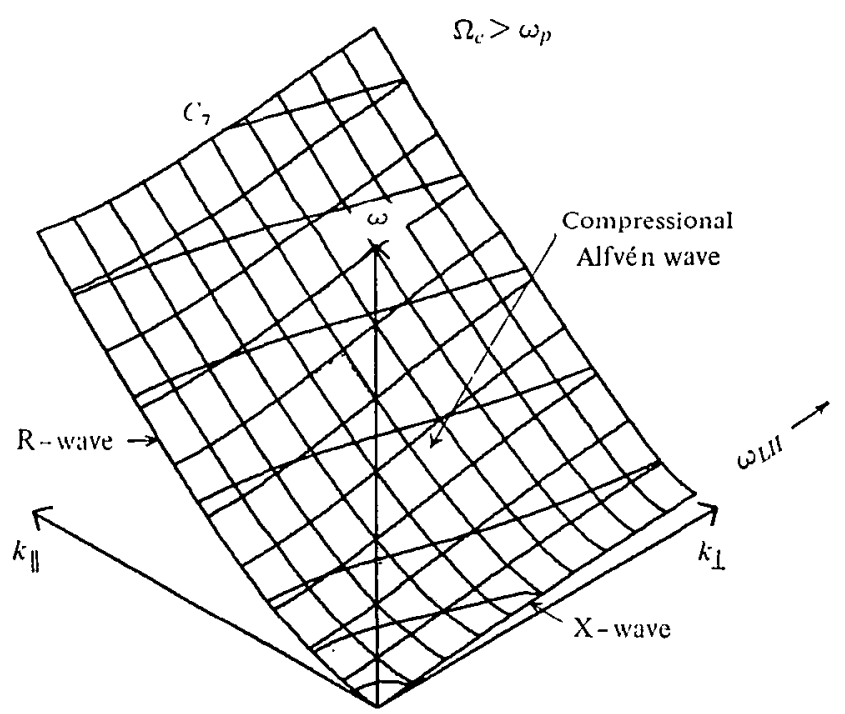

Figure 8. Compressional Alfvén-lower hybrid surface. $\omega v s$. $k_{1}$ and $k_{\perp}$. Perspective plot. Linear scale. $B_{0}=100 \mathrm{kG}, n_{\mathrm{a}}=10^{14} \mathrm{~cm}^{-3}, 0 \leqslant k_{1} \leqslant 5 \mathrm{~cm}^{-1}, 0 \leqslant k_{\perp} \leqslant 5 \mathrm{~cm}^{-1}, 0 \leqslant \omega \leqslant$ $1.15 \times 10^{11} \mathrm{sec}^{-1}$. Constant $\omega$ contours: $C_{1}=10^{9} \mathrm{sec}^{-1}, C_{2}=5 \times 10^{9} \mathrm{sec}^{-1}, C_{3}=1.257 \times 10^{10}$ $\mathrm{sec}^{-1}, C_{4}=3 \times 10^{10} \mathrm{sec}^{-1}, C_{5}=5 \times 10^{10} \mathrm{sec}^{-1}, C_{6}=7 \times 10^{10} \mathrm{sec}^{-1}, C_{7}=9 \times 10^{10} \mathrm{sec}^{-1}$. 


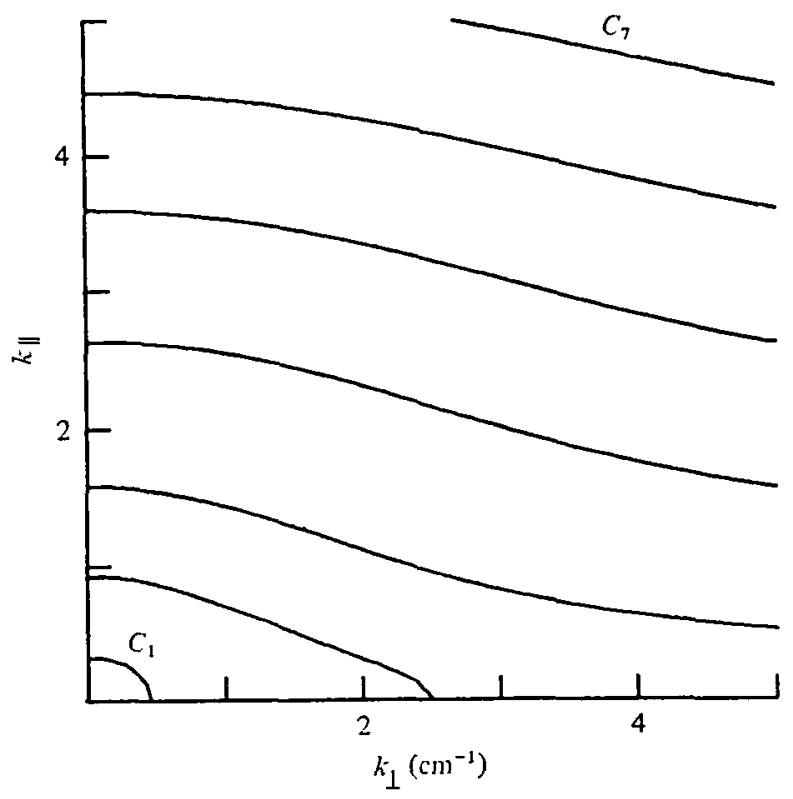

Figure 9. Contours of compressional Alfvén-lower hybrid surface. Contour values are the same as in figure 8 .

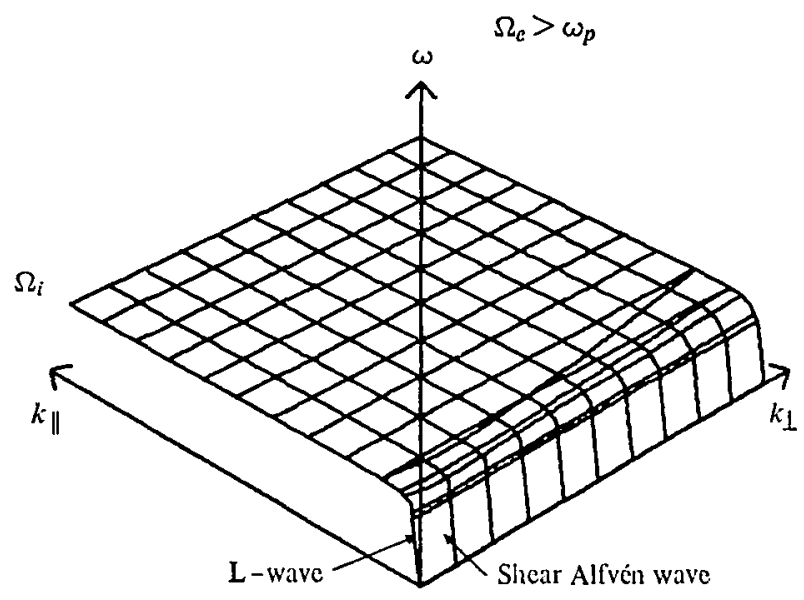

Frgure 10. Shear Alfvén-ion cyclotron surface. $\omega v s . k_{1}$ and $k_{\perp}$. Perspective plot. Linear scale. $B_{0}=100 \mathrm{kG}, n_{s}=10^{14} \mathrm{~cm}^{-3}, 0 \leqslant k_{1} \leqslant 50 \mathrm{~cm}^{-1}, 0 \leqslant k_{1} \leqslant 50 \mathrm{~cm}^{-1}, 0 \leqslant \omega \leqslant 5 \times 10^{9}$ $\mathrm{sec}^{-1}$. Contours $C_{1}=6.9 \times 10^{8} \mathrm{sec}^{-1}, C_{2}=7.5 \times 10^{8} \mathrm{sec}^{-1}, C_{3}=9 \times 10^{8} \mathrm{sec}^{-1}, C_{4}=9.3 \times 10^{8}$ $\sec ^{-1}, C_{5}=9.5 \times 10^{8} \mathrm{sec}^{-1}$.

$\theta_{r}$ asymptote. The phase velocity $(\omega / k)$ is zero at this angle of propagation.

Figure 8 expands the low frequency region of figure 6 . The absence of resonant angles $\left(\theta_{r}\right)$ for $\omega<\omega_{L H}$ is apparent from the surface shape. Figure 9 contains the associated contours. Such contours will not give rise to resonance cones which are possible for the $\omega>\omega_{L H}$ portion of this surface. 


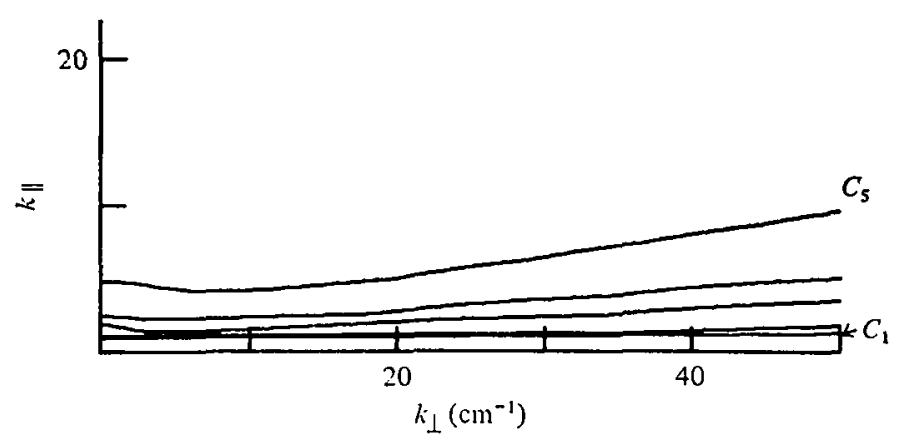

Figure 11. Contours of shear Alfvén-ion cyclotron surface. Contour values are the same as in figure 10 .

Figure 10 shows the surface which includes the shear Alfvén and ion cyclotron waves. We note that the surface is relatively insensitive to $k_{\perp}$ as suggested by the familiar approximate dispersion relation $\left(\omega=k_{\mathrm{n}} V_{A}\right.$, where $V_{A}$ is the Alfvén velocity). The surface disappears at $\theta=\pi / 2$, indicating the well-known result that the shear wave will not propagate perpendicular to a magnetic field. Along the field the wave is the left-hand circularly polarized wave $\left(n^{2}=L\right)$ which has a resonance at $\Omega_{i}$. Figure 11 is a contour plot for figure 10 .

\section{Group velocity}

Since the group velocity is given by $d \omega / d \mathbf{k}$ then it is evident that the direction of the group velocity at a point on a surface is the direction of steepest ascent and that the slope in this direction determines the magnitude of the group velocity. In figure 6 we see the possibility of backward waves which have components of group velocity and phase velocity perpendicular to the magnetic field in the opposite directions; note, however, that the phase and group velocity components are in the same direction along the field. Figure 10 shows that the shear wave group velocity lies predominantly along the magnetic field.

\section{Polar log plots}

Stringer used $\log \omega$ versus $\log k$ in presenting his results; in the remaining portion of this paper we will do the same; however, $k$ will be normalized to the minimum value of $k$ plotted. The group velocity for these surfaces is no longer simply the directional derivative on the surface; instead it is given by

$$
\mathbf{v}_{\mathbf{g r}}=v_{\mathrm{ph}}[(\partial \log \omega / \partial \log k) \hat{k}+(\partial \log \omega / \partial \theta) \hat{\theta}] .
$$

It should be noted that the quantity in the brackets is not the directional derivative on the polar log surface.

Figure 12 shows a superposition of the five surfaces. This is for the case of $\Omega_{c}>\omega_{p e}$ which corresponds to the linear plots discussed earlier. The range of values of $k$ is the same for all five surfaces. In the polar plots the value of $\log \omega$ 


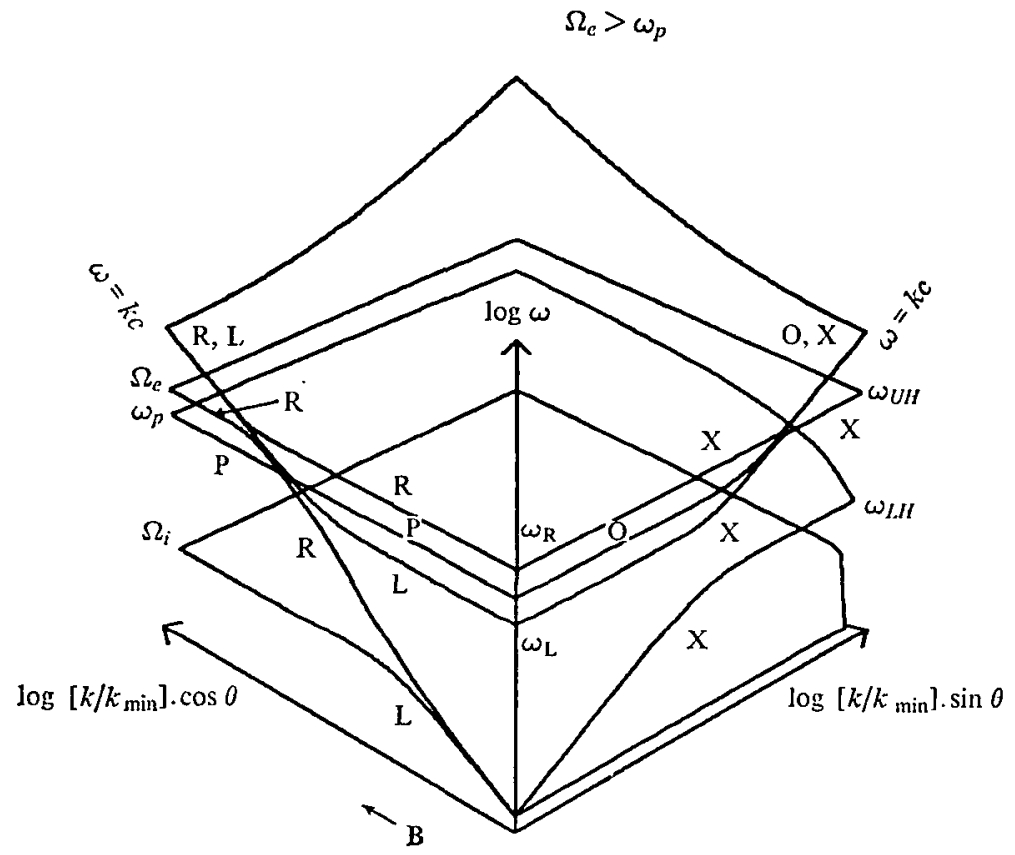

Figdre 12. Polar $\log$ plots [ $\log \omega$ vs. $\log \left(k / k_{\min }\right)$ and $\theta$ ]. Five surfaces $B=100 \mathrm{kG}, n_{e}=10^{14} \mathrm{~cm}^{-3}, 10^{-2} \leqslant k \leqslant 10^{3} \mathrm{~cm}^{-1}$.

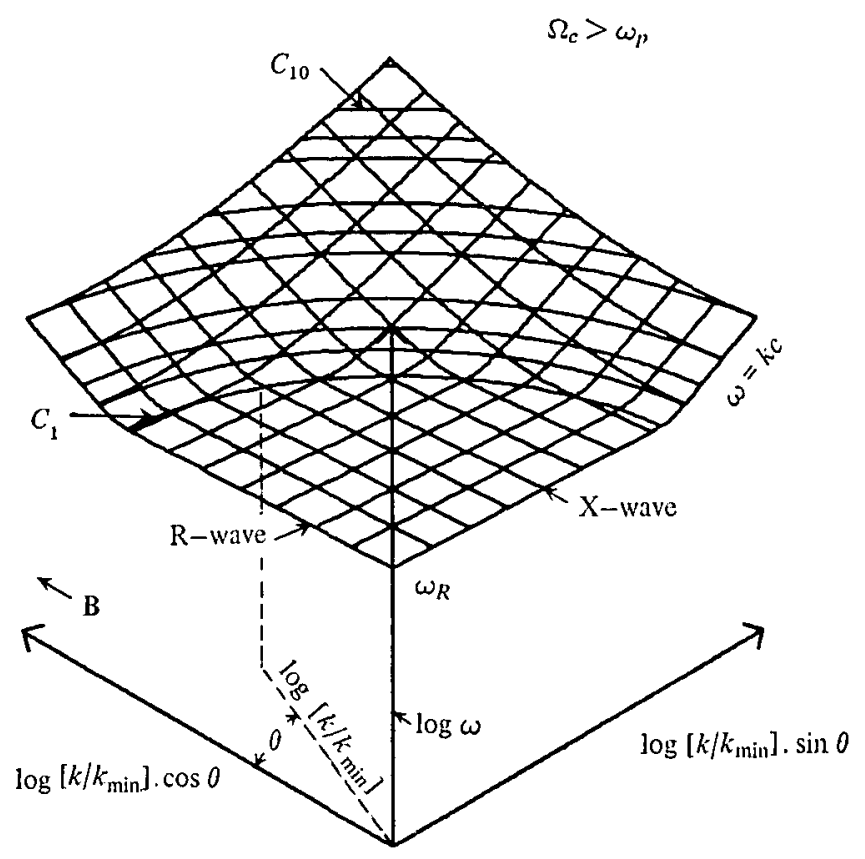

Figure 13. High frequency $R, X$ surface $\left[\log \omega v s \cdot \log \left(k / k_{\min }\right)\right.$ and $\left.\theta\right]$. $B=100 \mathrm{kG}, n_{s}=10^{14} \mathrm{~cm}^{-3}, 10^{-2} \leqslant k \leqslant 10^{3} \mathrm{~cm}^{-1}$. 


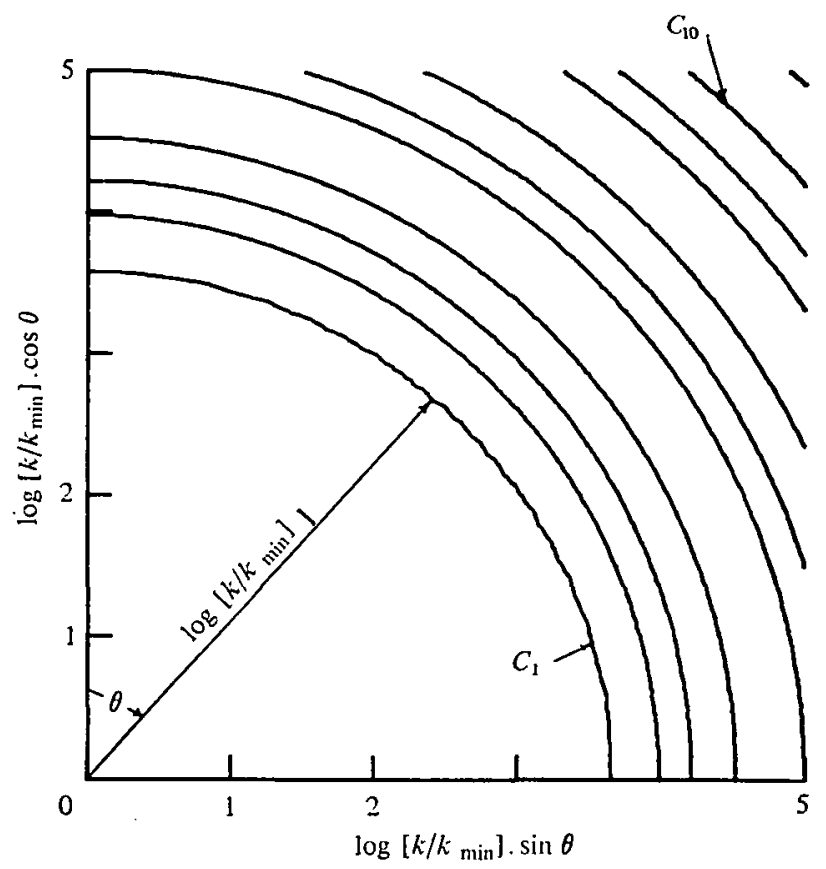

FraURe 14. Contour plots for figure 13. Contours: $C_{1,2,3}=2,3,5 \times 10^{12} \mathrm{sec}, C_{4,5,8}=1,3$, $5 \times 10^{13} \mathrm{sec}^{-1}, C_{78,8}=1,3,5 \times 10^{14} \mathrm{sec}^{-1}, O_{10,11}=1,3 \times 10^{15} \mathrm{sec}^{-1}$.

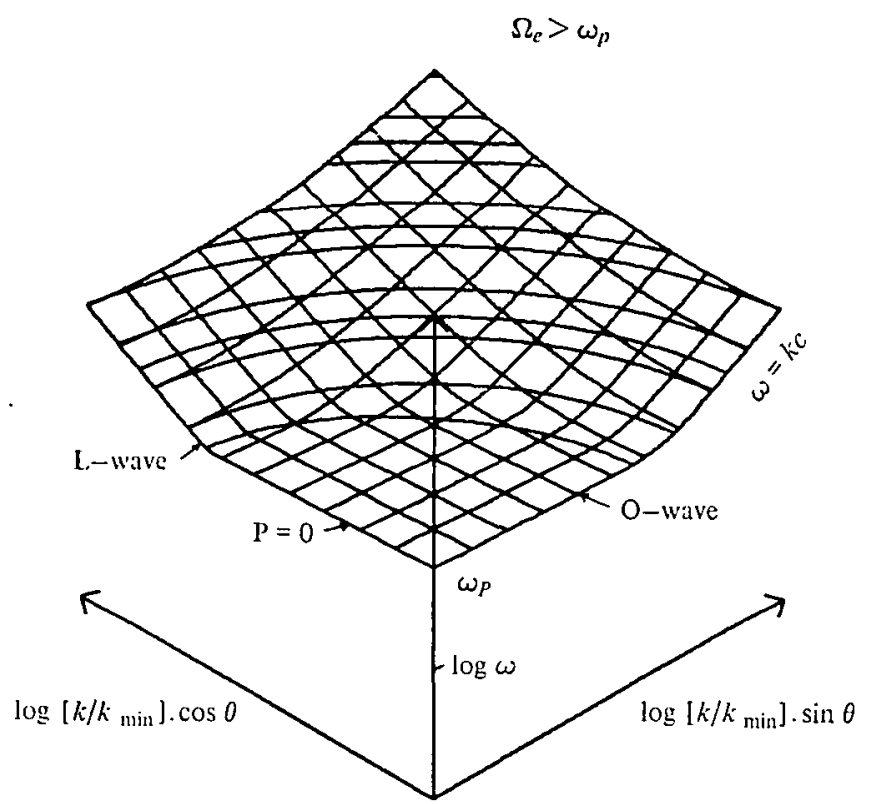

FIGURE 15. High frequency $L, O$ surface. Data are the same as in figure 13. 


$$
\Omega_{c}>\omega_{p}
$$

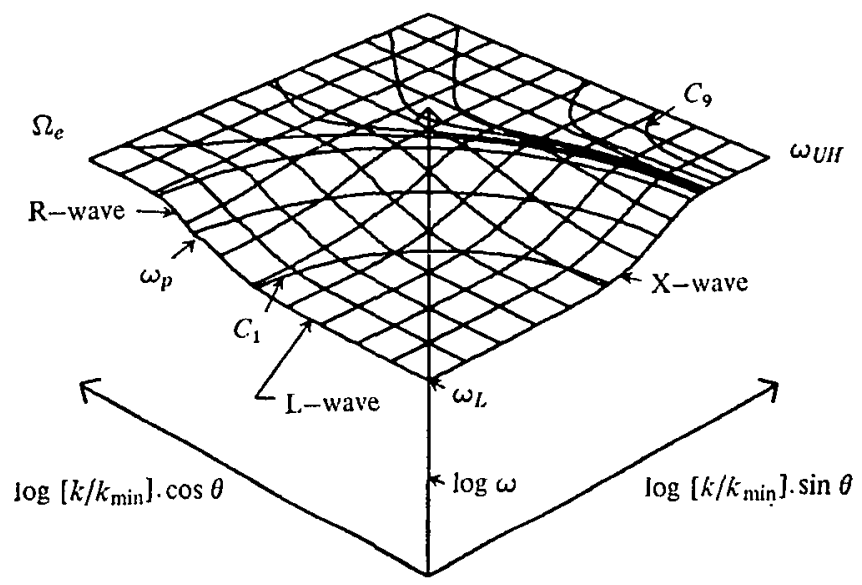

FIGURE 16. Electron cyclotron-upper hybrid surface. Data are the same as in figure 13.

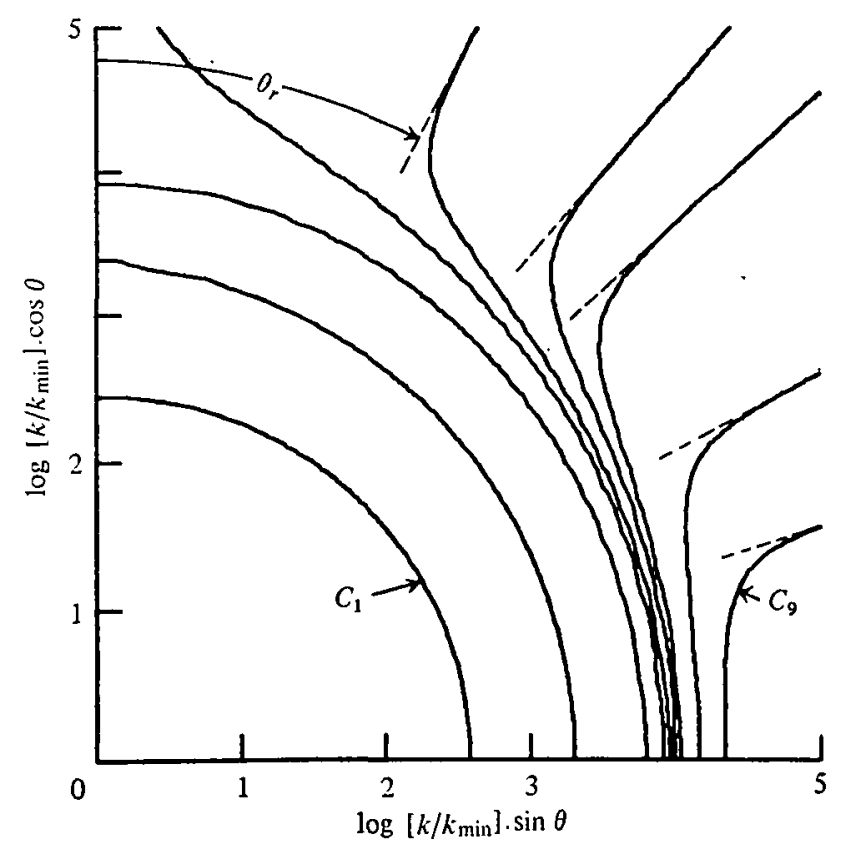

FIGURE 17. Contour plots of figure 16. $C_{1}=2 \times 10^{11} \mathrm{sec}^{-1}, C_{2}=6 \times 10^{11} \mathrm{sec}^{-1}, C_{3}=1.6 \times 10^{12}$ $\mathrm{sec}^{-1}, C_{4}=1.759 \times 10^{12} \mathrm{sec}^{-1}, C_{5}=1.788 \times 10^{12} \mathrm{sec}^{-1}, C_{6}=1.8 \times 10^{12} \mathrm{sec}^{-1}, C_{7}=1.81 \times 10^{12}$ $\mathrm{sec}^{-1}, C_{8}=1.83 \times 10^{12} \mathrm{sec}^{-1}, C_{9}=1.84 \times 10^{12} \mathrm{sec}^{-1}$. 


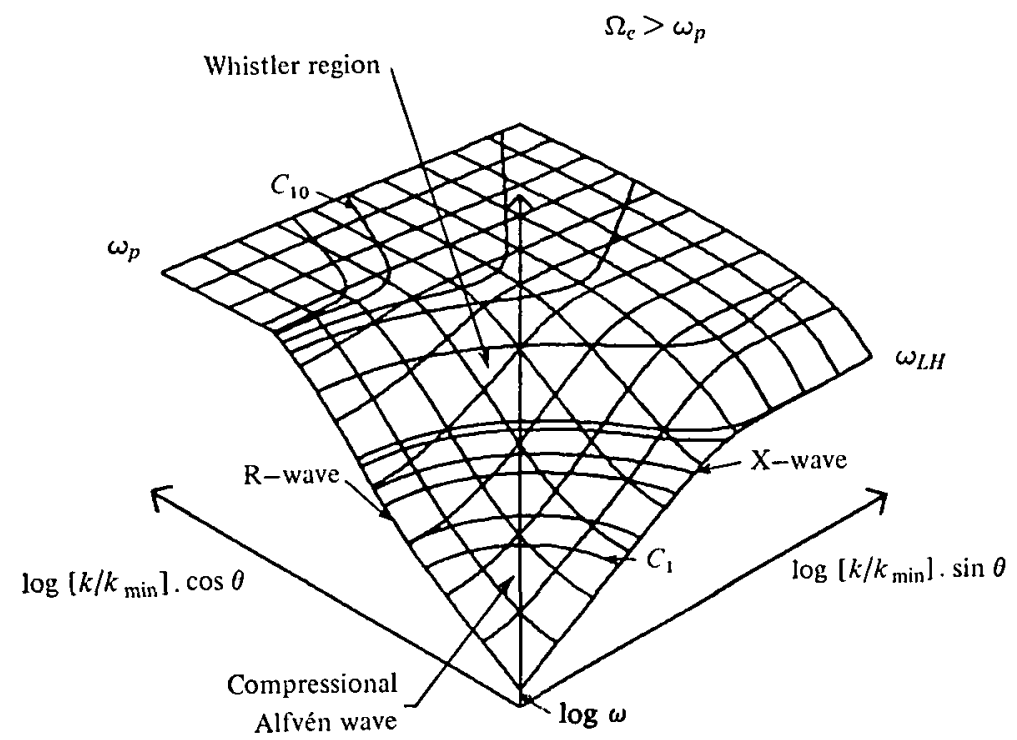

FiquRe 18. Compressional Alfvén-lower hybrid surface. Data are the same as in figure 13.

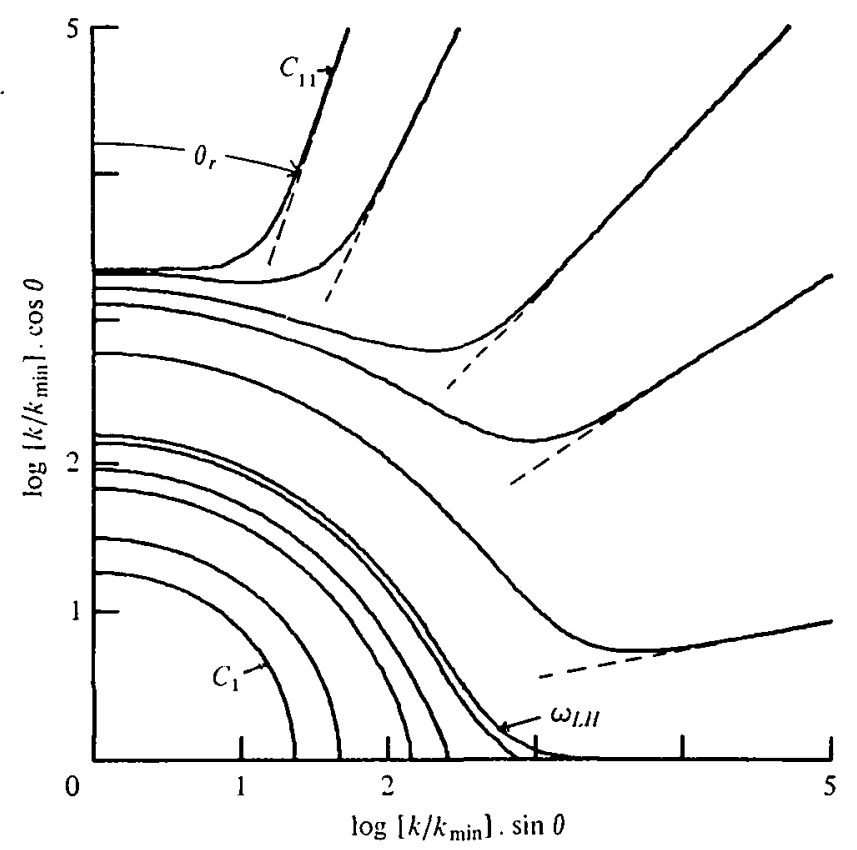

Figure 19. Contour plots of figure 18. $C_{1}=5 \times 10^{8} \mathrm{sec}^{-1}, C_{2,3,4}=1,3,6 \times 10^{9} \mathrm{sec}^{-1}, C_{5,6}=1$, $1 \cdot 257 \times 10^{10} \mathrm{sec}^{-1}, C_{7,8,9,10,11}=1,3,4,5,5 \cdot 3 \times 10^{11} \mathrm{sec}^{-1}$. 


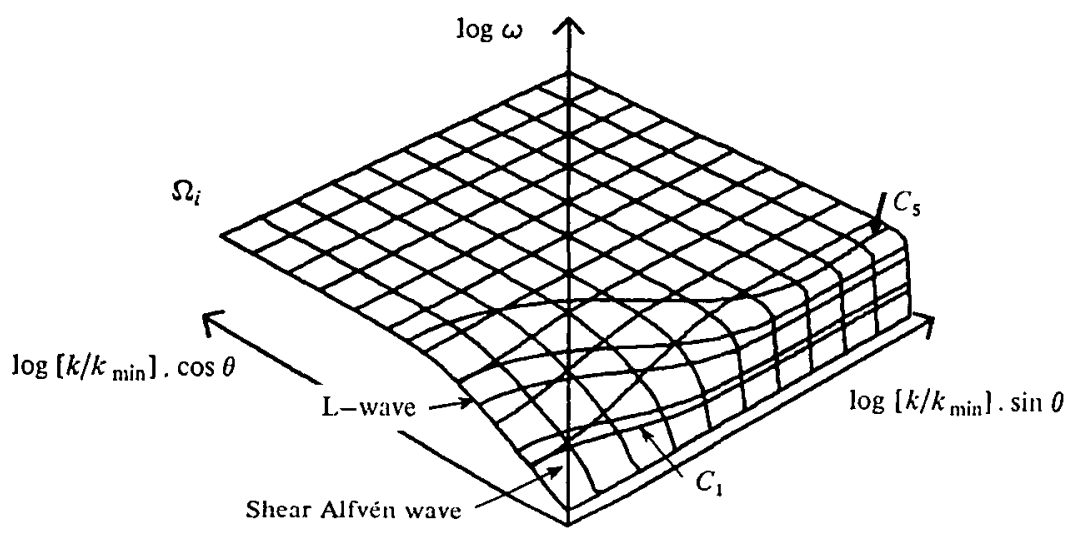

Fraure 20. Shear Alfvén-ion cyclotron surface.

Data are the same as in figure 13.

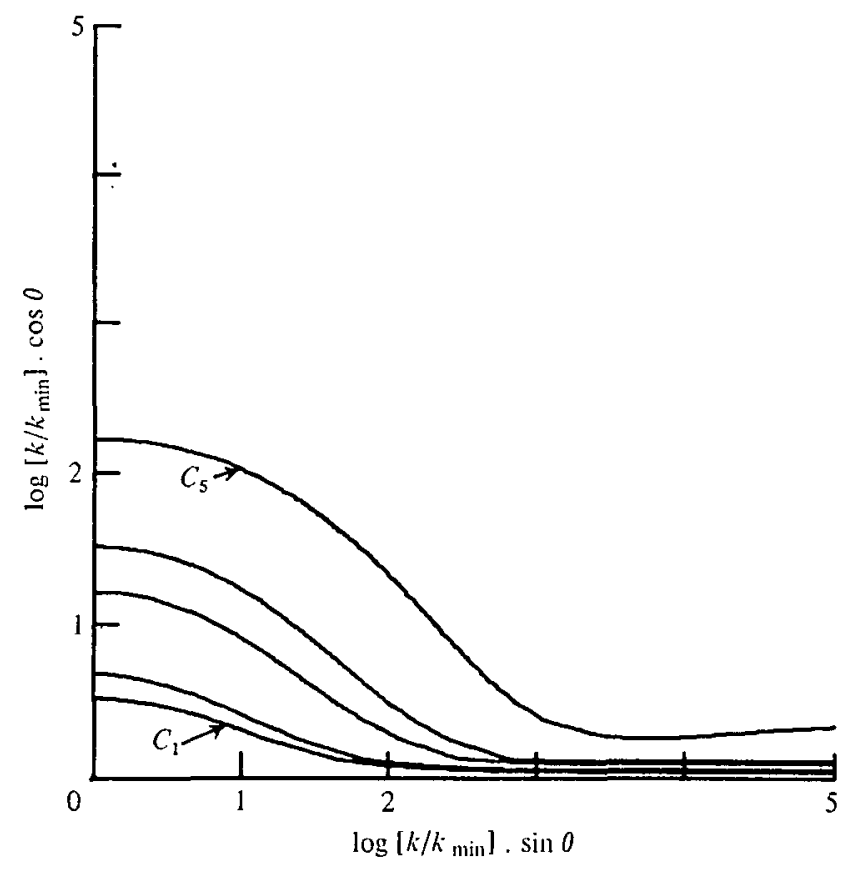

FIGURe 21. Contour plots of figure 20. $C_{1}=7 \times 10^{7} \mathrm{sec}^{-1}$, $C_{2,3,4,5}=1,3,5,8 \times 10^{8} \mathrm{sec}, 8.7 \times 10^{8} \mathrm{sec}^{-1}$.

at the origin is found by substituting $k=0$ in the dispersion relation. Difficulties that arise at the origin with the lower two surfaces are removed by arbitrarily taking $\omega=k_{\min } V_{A}$ for both surfaces at this point. It is important to remember that the axes in these plots are not $k_{\perp}$ and $k_{\|}$. A constant $\theta$ cut through a surface produces a Stringer dispersion curve. Figures 13-21 show individually the five 


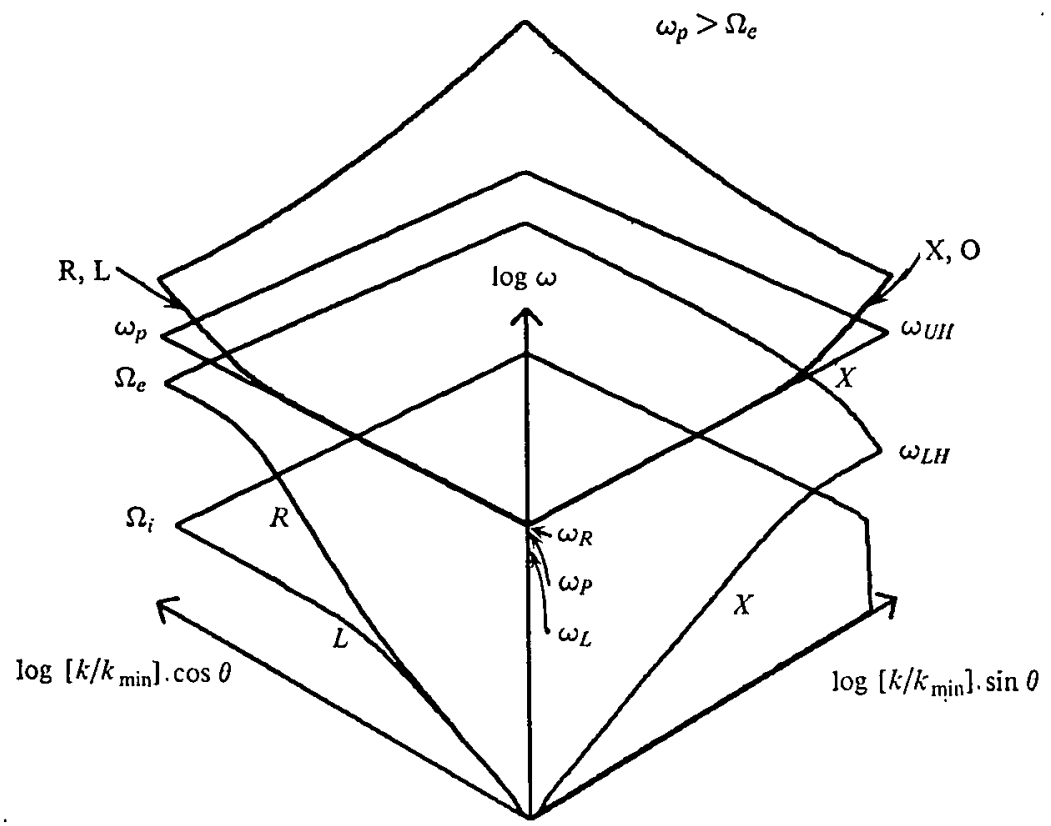

FIGURE 22. Five surfaces-polar $\log$ perspective. $\omega_{p}>\Omega_{b}\left[B=10 \mathrm{kG}, n_{b}=10^{15} \mathrm{~cm}^{-3}\right]$, $10^{-2} \leqslant k<10^{3} \mathrm{~cm}^{-1}$.

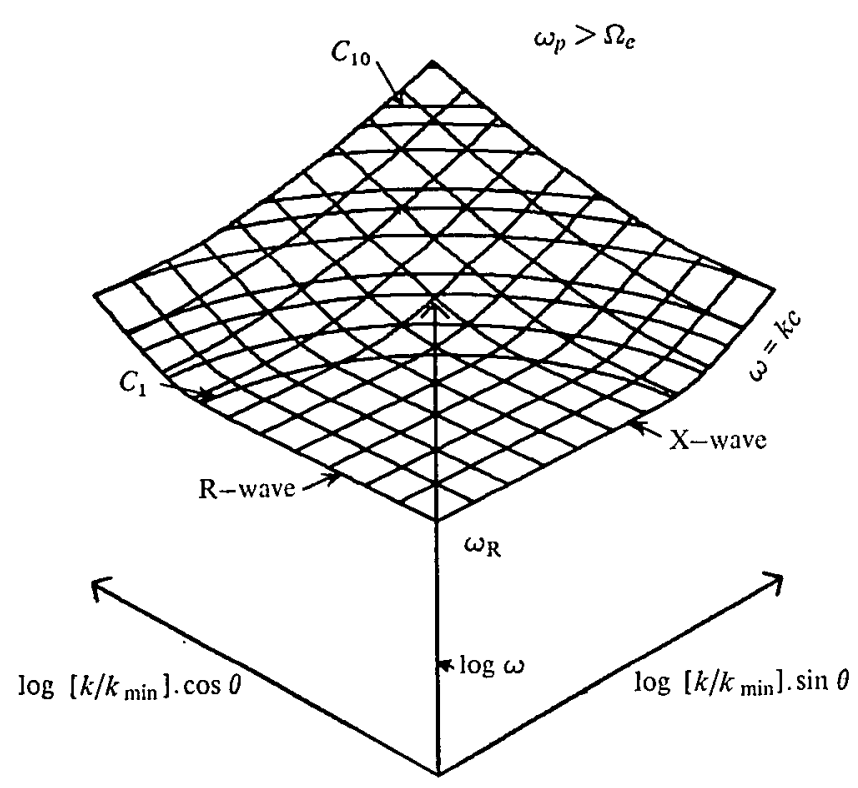

Figdre 23. High frequency $R, X$ surface. Data are the same as in figure 22 . 


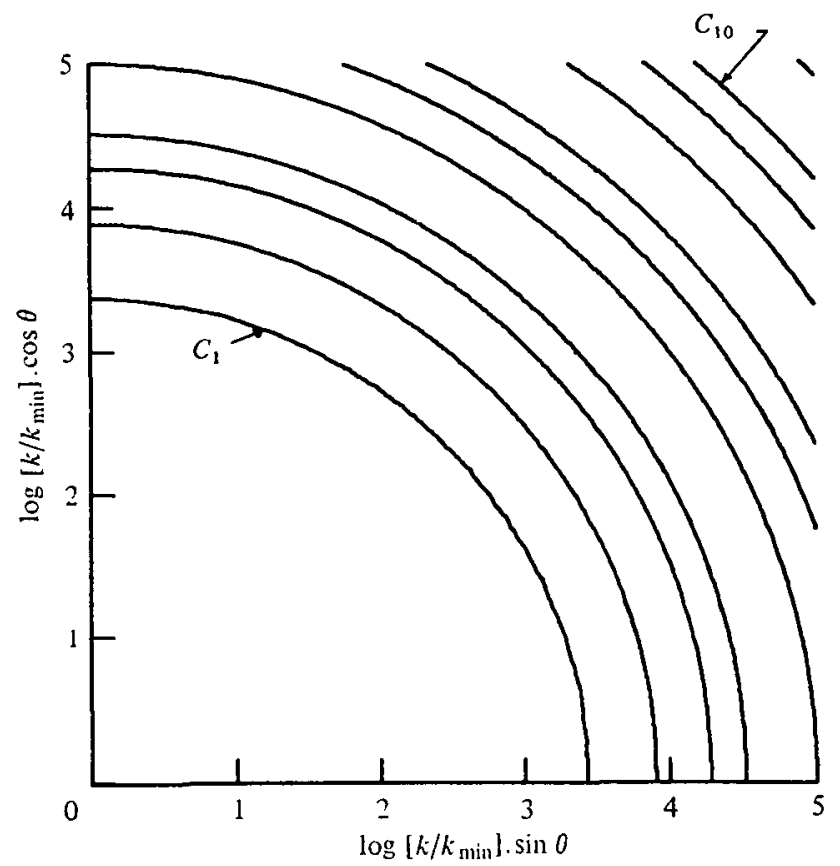

Fraure 24. Contour plot of figure 23. Contour $C_{1,2,3}=2,3,6 \times 10^{12} \mathrm{sec}^{-1}, C_{4,8,8}=1 \cdot 3$, $6 \times 10^{13} \mathrm{sec}^{-1}, C_{7,8,9}=1,3,6 \times 10^{14} \mathrm{sec}^{-1}, C_{10,11}=1,3 \times 10^{15} \mathrm{sec}^{-1}$.

$$
\omega_{p}>\Omega_{e}
$$

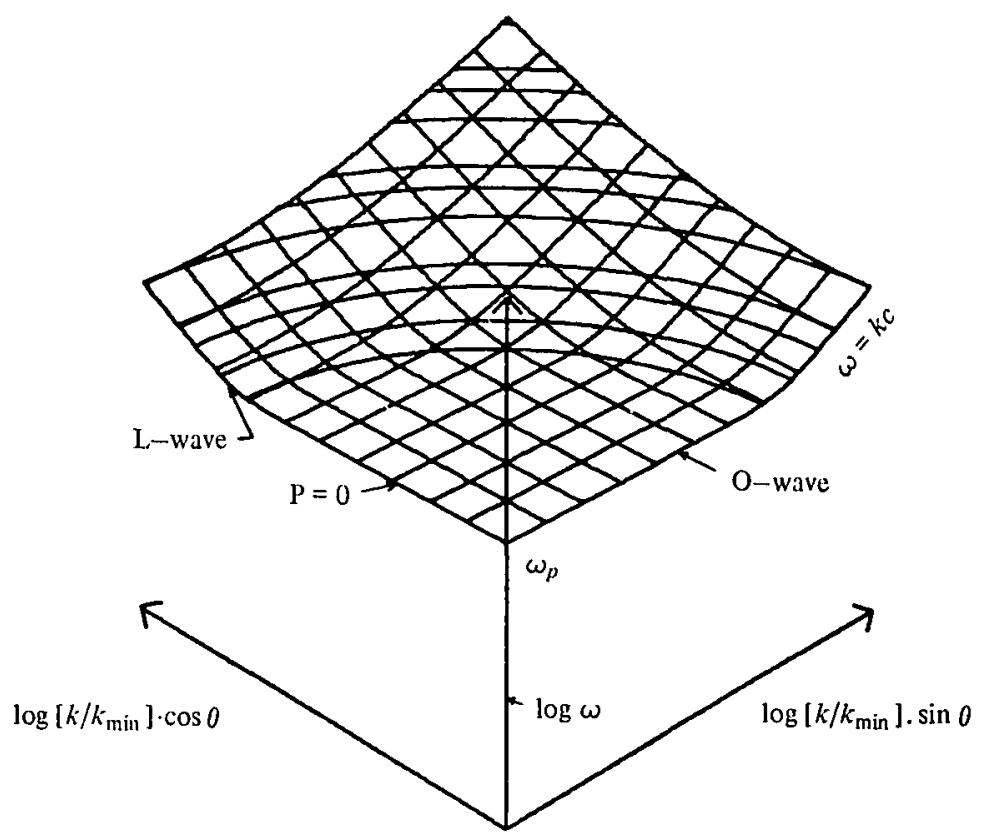

Fraure 25. High frequency $L, O$ surface. Data are the same as in figure 22 . 


$$
\omega_{p}>\Omega_{c}
$$

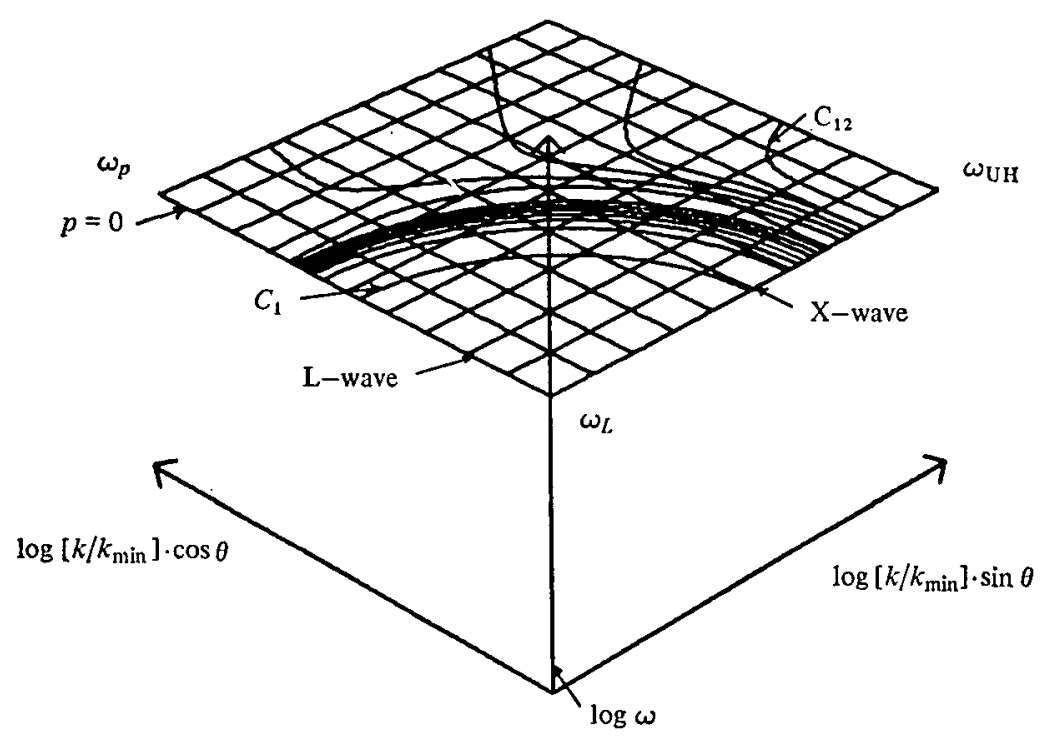

Fiadre 26. $L$-wave and upper hybrid surface.

Data are the same as in figure 22.

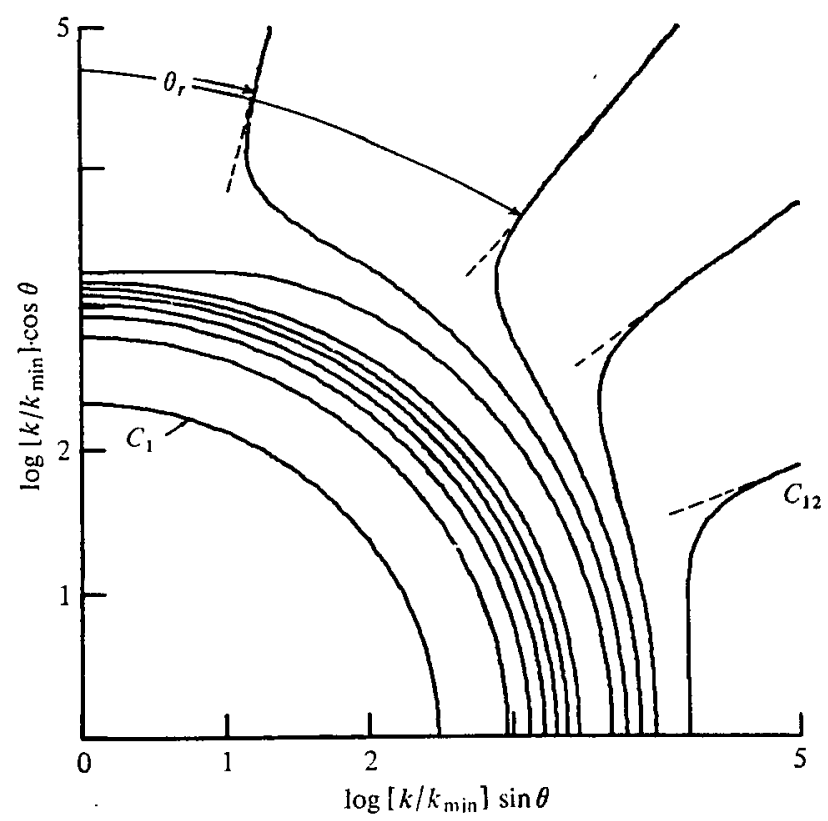

FraURE 27. Contour plotfor figure 26. Contours $C_{1}=1.7 \times 10^{12} \mathrm{sec}^{-1}, C_{2-7}=1 \cdot 71-1.76 \times 10^{12}$ $\sec ^{-1}\left(\Delta \omega=10^{10} \mathrm{sec}^{-1}\right.$ in equal steps $), C_{8}=1.78 \times 10^{12} \mathrm{sec}^{-1}, C_{9}=1.785 \times 10^{12} \mathrm{sec}^{-1}, C_{10}=$ $1.788 \times 10^{12} \mathrm{sec}^{-1}, C_{11}=1.79 \times 10^{12} \mathrm{sec}^{-1}, C_{12}=1.792 \times 10^{12} \mathrm{sec}^{-1}$. 


$$
\omega_{p}>\Omega_{e}
$$

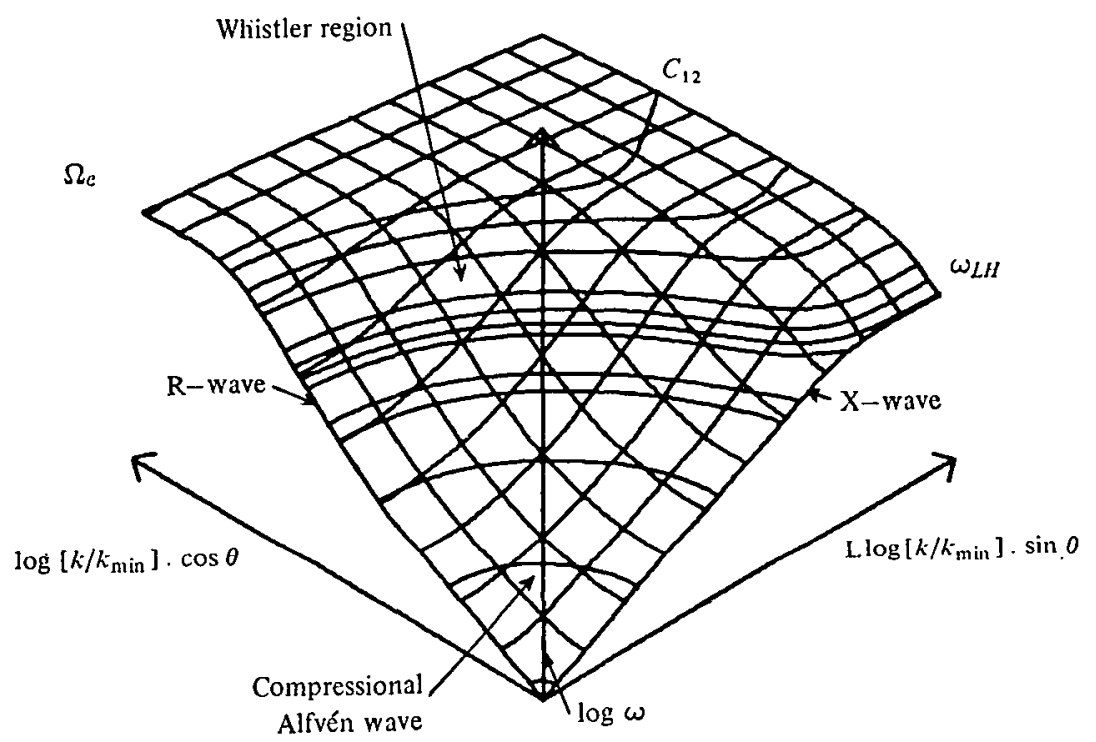

FIGURE 28. Electron cyclotron-compressional Alfvén-lower hybrid surface. Data are the same as in figure 22.

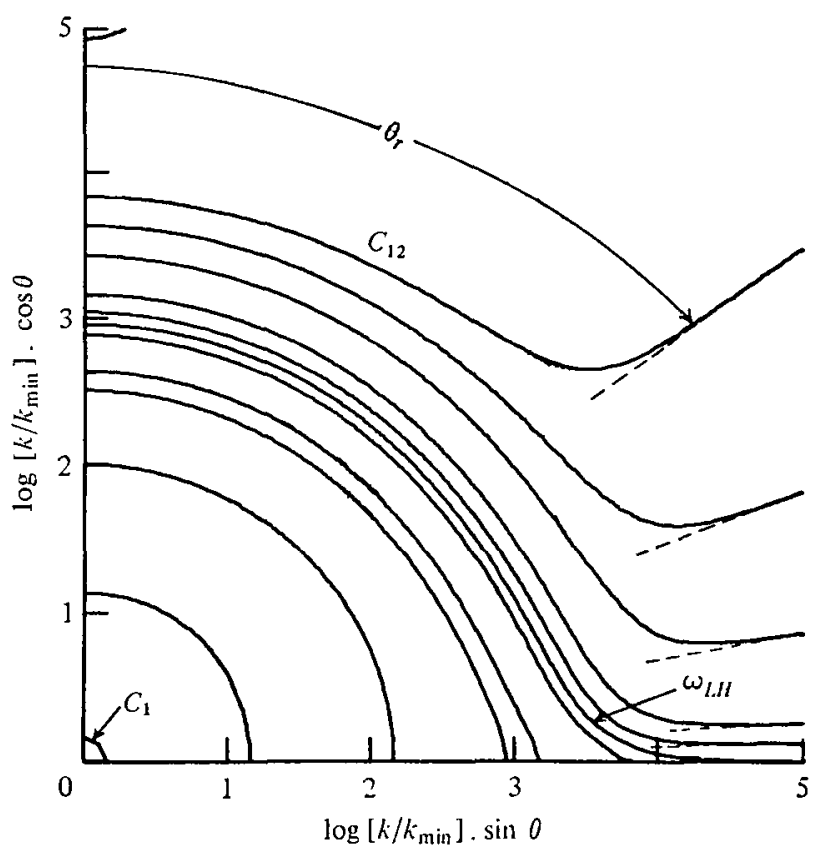

Figure 29. Contour plot for figure 28. $C_{1}=10^{6} \mathrm{sec}^{-1}, C_{2}=10^{7} \mathrm{sec}^{-1}, C_{3,4}=1,6 \times 10^{8} \mathrm{sec}^{-1}$, $C_{5,6,7,8}=1,3,4,6 \times 10^{9} \mathrm{sec}^{-1}, C_{9,10,11}=1,3,6 \times 10^{10} \mathrm{sec}^{-1}, C_{18,13}=1,1.75 \times 10^{11} \mathrm{sec}^{-1}$. 
$\omega_{p}>\Omega_{c}$

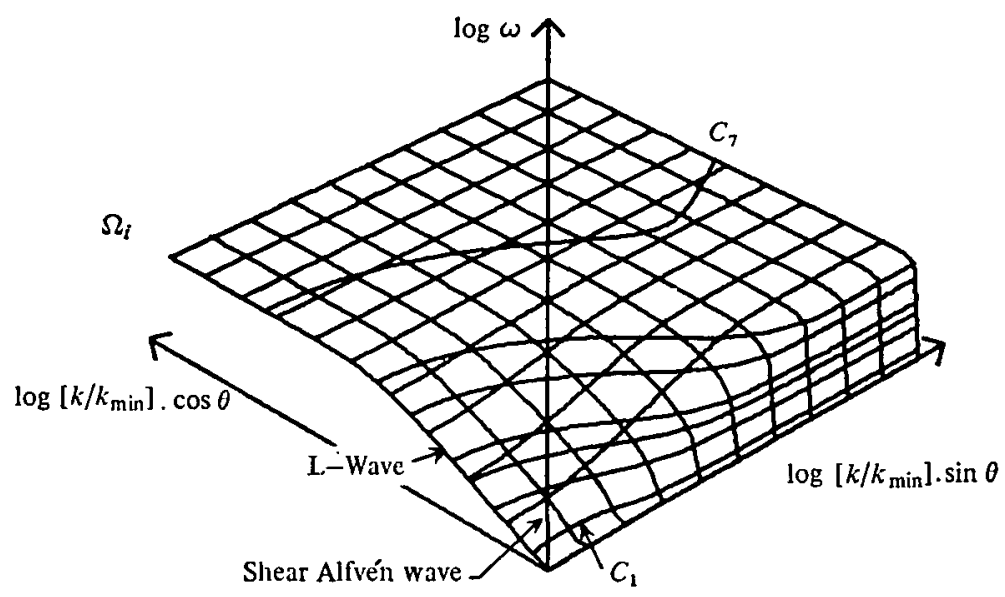

Fraure 30. Shear Alfvén-ion cyclotron surface. Data are the same as in figure 22.

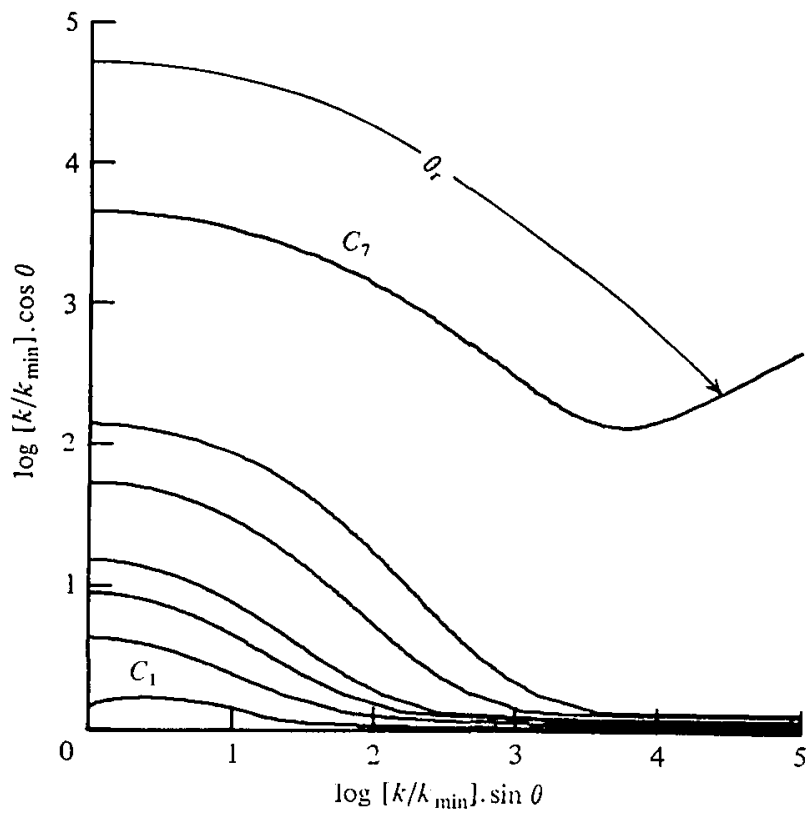

Figure 31. Contour plot for figure 30. Contours $C_{1,2,3}=1,3,6 \times 10^{3} \mathrm{sec}^{-1}$, $C_{4,5,6}=1,3,6 \times 10^{7} \mathrm{sec}^{-1}, C_{7}=9.57 \times 10^{7} \mathrm{sec}^{-1}$.

surfaces and their contours. The discussion of the linear plots applies to these surfaces.

Figure 22 shows five surfaces for a high density case $\left(\omega_{p}>\Omega_{e}\right)$. We note in this case that the compressional Alfven-lower hybrid surface now includes the electron cyclotron wave. The upper hybrid surface now contains the plasma oscillations along the magnetic field direction. The individual surfaces and their 
contours are shown in figures 23-31. The labelling of the surfaces should be sufficient explanation of the wave features.

Complete coverage of all cases included in the CMA diagram requires surfaces with conditions such that $\omega_{p e}<\Omega_{i}$. The conditions for this case usually occur only in the extremely thin low-density layer at the plasma surface; for this reason the surfaces are omitted from this paper. The inclusion of thermal modes will be the subject of a future paper.

This work was supported by the Texas Atomic Energy Research Foundation.

\section{REFERENCES}

Alus, W. P. 1959 Sherwood Conference on Controlled Fusion, Gatlinburg, p. 32, TID.7582. Aulis, W. P., Buchsbaum, S. J. \& Bers, A. 1962 Waves in Plasmas. Wiley.

Clemmow, P. C. \& Moliady, R. F. 1955 Physics of the Ionosphere, p. 340. Physical Society, London.

Stix, T. H. 1962 The Theory of Plasma Waves. McGraw-Hill.

Stringer, T. E. 1963 J. Nucl. Energy, Part O, 5, 89. 